\title{
ULTRA-SONOGRAFIA TRIDIMENSIONAL DO COLO UTERINO NA GESTAÇÃO: PERSPECTIVAS*
}

\author{
Rosieny Souza Brandão ${ }^{1}$, Carlos Geraldo Viana Murta ${ }^{2}$, Antonio Fernandes Moron ${ }^{3}$, \\ Rosiane Mattar ${ }^{4}$, Claudio Rodrigues Pires ${ }^{5}$, Eduardo Almeida Guerzet ${ }^{6}$
}

\begin{abstract}
Resumo A medida do comprimento cervical por ultra-sonografia transvaginal é útil no rastreamento do parto prematuro, sendo o encurtamento do colo fator preditor do trabalho de parto pré-termo. Os métodos tradicionais para avaliar a cérvice na gestação são limitados e insatisfatórios. 0 exame de toque digital, considerado método padrão, demonstra variação entre diferentes examinadores, entretanto, a ultra-sonografia transvaginal é exame eficiente durante a gravidez. Recentemente, o exame ultra-sonográfico tridimensional tem sido utilizado na prática clínica, incluindo o estudo do colo. Grande volume de informações pode ser obtido e armazenado utilizando-se a tecnologia tridimensional. A informação armazenada permite ser manipulada e analisada por número infinito de planos. $O$ exame ultra-sonográfico transvaginal tridimensional é o único capaz de obter o plano coronal pela visualização da imagem em organização multiplanar. Este método aparenta oferecer potencial diagnóstico no aumento da acurácia da ultra-sonografia cervical.
\end{abstract}

Unitermos: Colo uterino; Gravidez; Ultra-sonografia tridimensional.

Abstract Three-dimensional ultrasound of uterine cervix in pregnancy: prospects.

The measurement of cervical length utilizing transvaginal ultrasound is of help for pregnant women screening for premature labor, the shortening of the cervix being a predictor of higher risk of preterm delivery. Traditional methods for evaluating the uterine cervix during pregnancy are limited and unsatisfactory. Digital examination of cervix, considered as the standard method, demonstrates large variation among different examiners; on the other hand, transvaginal sonography is an effective method of examination during pregnancy. Recently, three-dimensional ultrasound has been used in the clinical practice including the study of uterine cervix. The three-dimensional technology allows acquisition and storage of a large volume of data. Such stored information can be reformatted and analyzed through an unlimited number of planes. The threedimensional transvaginal ultrasound is the unique imaging method capable of obtaining a true coronal plane by direct correlation of the views in a multiplanar display. This method seems to offer a diagnostic potential because of the cervical ultrasonography increased accuracy.

Keywords: Uterine cervix; Pregnancy; Three-dimensional ultrasonography.

\section{INTRODUÇÃO}

O desenvolvimento da ultra-sonografia tridimensional $^{(\mathbf{1})}$ (US 3D) foi proposto em

* Trabalho realizado no Departamento de Obstetrícia, Disciplina de Medicina Fetal da Universidade Federal de São PauloEscola Paulista de Medicina, São Paulo, SP, e no Centro de UItra-Sonografia Capixaba (MedfetUS), Vitória, ES.

1. Mestre pela Universidade Federal de São Paulo-Escola Paulista de Medicina, Médica Ultra-sonografista do CDI - Centro de Diagnóstico por Imagem, Vitória/ES.

2. Professor Doutor do Departamento de Ginecologia e Obstetrícia da Universidade Federal do Espírito Santo.

3. Professor Doutor Titular do Departamento de Obstetrícia da Universidade Federal de São Paulo-Escola Paulista de Medicina.

4. Professora Doutora Adjunta do Departamento de Obstetrícia, Coordenadora do Programa de Pós-graduação da Universidade Federal de São Paulo-Escola Paulista de Medicina.

5. Professor Doutor pela Universidade Federal de São PauloEscola Paulista de Medicina, Médico Diretor do Cetrus - Centro de Treinamento de Ultra-Sonografia, São Paulo, SP.

6. Professor Doutor Adjunto do Departamento de Tocoginecologia da Escola de Medicina da Santa Casa de Misericórdia de Vitória, ES.

Endereço para correspondência: Dra. Rosieny Souza Brandão. Avenida Champagnat, 501, sala 403, Praia da Costa. Vila VeIha, ES, 29100-010. E-mail: rosienysbrandao@terra.com.br

Recebido para publicação em 30/7/2004. Aceito, após revisão, em 22/2/2006.
1950. O Voluson 350, primeiro aparelho de ultra-som tridimensional comercialmente disponível, foi apresentado no congresso internacional de radiologia em Paris, em $1989^{(2)}$. No entanto, o grande avanço da tecnologia tridimensional, em especial na ginecologia e na obstetrícia, ocorreu no final dos anos 80, após o desenvolvimento dos sistemas de computação, principalmente da computação gráfica ${ }^{(3)}$.

Desde a década de 70, a US tem sido utilizada na avaliação do colo uterino na gestação, identificando possíveis modificações estruturais da cérvice no transcorrer da gravidez ${ }^{(4,5)}$. Segundo alguns pesquisadores, o risco de parto prematuro é inversamente proporcional à medida do comprimento cervical aferido por US endovaginal $^{(6-11)}$.

Com o intuito de aprimorar o estudo do colo uterino por imagem, Hoesli et al. ${ }^{(\mathbf{1 2})}$, em 1999, em pesquisa inédita, publicaram resultados referentes à aplicação da US 3D na análise do colo uterino em gestantes.

A seguir, outros autores avaliaram a medida do comprimento da cérvice com a tecnologia tridimensional, comparando-a com a US bidimensional (US 2D), mostrando a supremacia da US 3D em relação à US 2D no estudo da morfologia cervical ${ }^{(\mathbf{1 3})}$, e a existência de diferenças entre as medidas do comprimento do colo aferidas por exames bidimensional e tridimensional ${ }^{(\mathbf{1 4})}$.

A crescente preocupação com a morbidade e a mortalidade neonatal vinculadas à prematuridade, o conhecimento das transformações cervicais na gravidez, e a possibilidade de aprimorar a avaliação sonográfica do colo uterino, pela utilização da técnica tridimensional, para a prevenção do trabalho de parto prematuro (TPP), motivaram a realização deste trabalho, que vislumbra a perspectiva do emprego da US 3D no incremento da avaliação cervical. 


\section{US 3D DO COLO UTERINO NA GESTAÇÃO}

No final da década de 90, a US 3D foi introduzida na gestação para estudo do colo uterino no rastreamento do $\mathrm{TPP}^{(\mathbf{1 2})}$. As primeiras pesquisas relacionavam a medida do volume da cérvice aferido via vaginal ${ }^{(12)}$ e/ou abdominal ${ }^{(15,16)}$ com a biometria cervical no rastreamento do trabalho de parto pré-termo.

Os resultados desses estudos evidenciaram boa correlação entre a US 2D e a US 3D na aferição do comprimento e volume da cérvice, respectivamente ${ }^{(12,15)}$. No entanto, a medida do comprimento do colo foi superior à do volume, na detecção de mulheres de risco para parto prematuro ${ }^{(12)}$.

Por outro lado, o valor preditivo positivo (VPP) da análise do volume cervical aferido pela US $3 \mathrm{D}$ em gestantes com colo uterino curto $(\leq 26 \mathrm{~mm})$ foi maior do que o VPP encontrado na medida do comprimento da cérvice obtida por US 2D, identificando com precisão as mulheres que evoluiriam para parto pré-termo ${ }^{(17)}$.

Bega et al. ${ }^{(18)}$,em 2000, foram os primeiros pesquisadores que utilizaram o exame tridimensional endovaginal na aferição do comprimento do colo uterino na gravidez, juntamente com a US 2D, comparando ambos os métodos. Foram avaliadas as gestantes entre 11 e 32 semanas de gestação (total de 37 exames) e foram aferidas as medidas do comprimento e/ou afunilamento cervical por exame bidimensional no plano sagital e pelo tridimensional em exposição multiplanar, nos planos sagital e coronal ${ }^{(18)}$. Vale ressaltar que a organização multiplanar (Figura 1), própria do sistema tridimensional, possibilita visão simultânea dos três cortes distintos do colo: sagital, transverso e axial ${ }^{(18)}$

Segundo Baga et al. ${ }^{(\mathbf{1 8 )}}$, a US 2D foi ineficaz em definir o verdadeiro plano sagital do colo em número importante de exames, visto que detectaram diferenças entre 5 e $15 \mathrm{~mm}$ nas medidas do comprimento cervical aferidas pelos diferentes métodos. No entanto, na comparação das médias das medidas, esses pesquisadores evidenciaram diferença estatisticamente significante entre as medidas do diâmetro do funil cervical aferidas no plano sagital no exame bidimensional e coronal no tri-

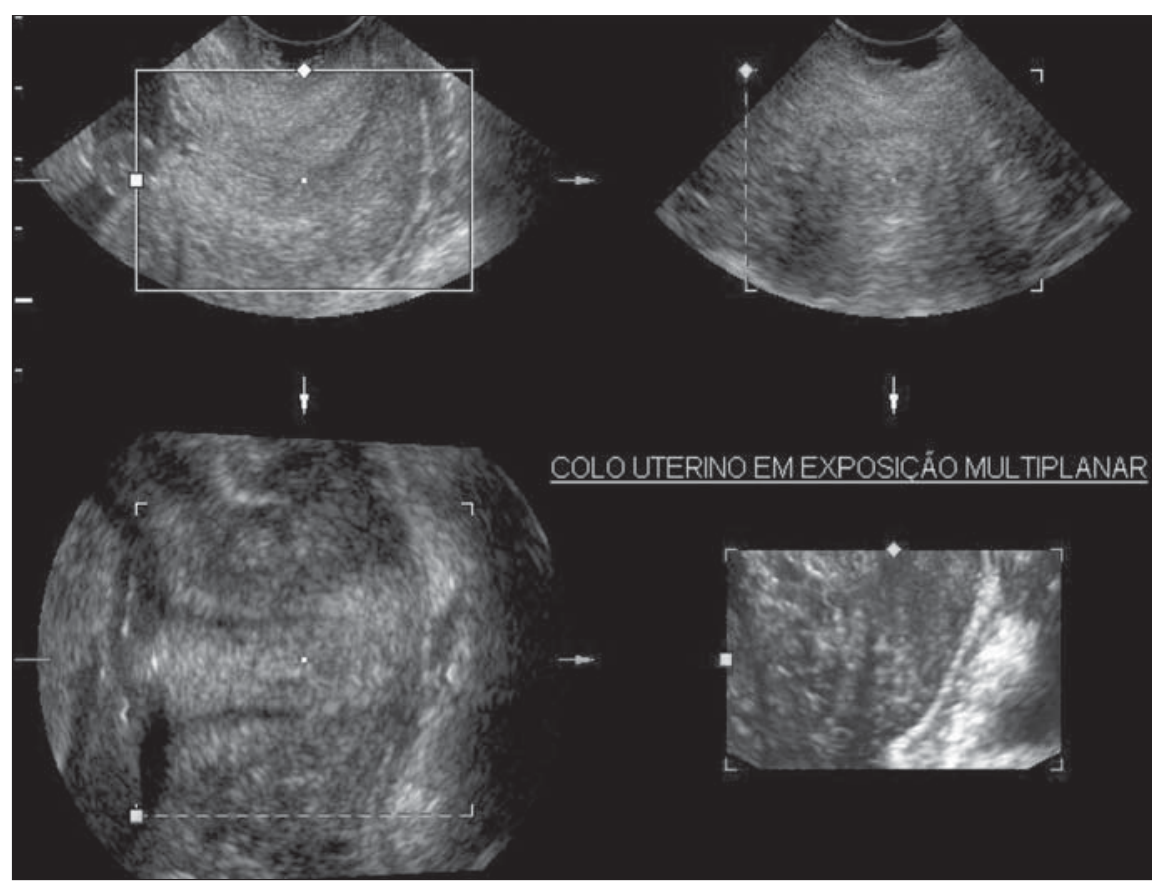

Figura 1. Imagem ultra-sonográfica tridimensional do colo uterino em exposição multiplanar exibindo simultaneamente os planos sagital, axial e coronal.

dimensional, e na US 3D entre os planos sagital e coronal. Relataram que a US 2D evidenciou limitação técnica na avaliação do real comprimento cervical devido à dificuldade de mobilização da sonda no canal vaginal, resultando corte meio oblíquo do colo no plano sagital, principalmente quando a cérvice se encontrava inclinada ou curvada. Em relação ao afunilamento, a US 3D detectou sua presença em número de casos maior que a US convencional, revelando o fato de que em algumas ocasiões a dilatação do orifício interno do colo se apresentava de maneira assimétrica em forma de fenda ou achatada, sendo sua identificação favorecida pelo corte coronal do colo exibido no exame tridimensional $^{(18)}$. Salienta-se que a reprodução e a avaliação da imagem em corte coronal são exclusivas da tecnologia tridimensional ${ }^{(\mathbf{1 8 )}}$. Bega et al. concluíram que a US 3D favorece o estudo mais detalhado da anatomia e biometria cervical que a US 2D.

Pesquisando o mesmo tema, em 2003, Severi et al. ${ }^{(\mathbf{1 3 )}}$ realizaram estudo semelhante ao de Bega et al., examinando 103 grávidas da população geral no segundo e no terceiro trimestre de gestação, buscando melhorar o valor preditivo do exame ecográfico no rastreamento do TPP. Severi et $a l$. Mencionaram, em seus resultados, que houve diferença $\geq 5 \mathrm{~mm}$ entre as medidas da cérvice aferidas nas diferentes técnicas no corte sagital em 48,5\% dos casos, sendo a medida do comprimento do colo maior no exame tridimensional do que no bidimensional $^{(13)}$. Em relação à comparação das médias das medidas do comprimento cervical, esses estudiosos relataram diferença significante entre os métodos ${ }^{(13)}$.

Concernente ao afunilamento, a US 3D diagnosticou dilatação do orifício interno do colo em número maior de mulheres que a US 2D, atribuindo a superioridade da técnica tridimensional à possibilidade de visualização da cérvice no corte coronal ${ }^{(13)}$. De acordo com Severi et al. ${ }^{(13)}$, o estudo do colo uterino obteve êxito com o emprego da tecnologia tridimensional devido à exposição das imagens em organização multiplanar, salientando-se a notória importância do corte coronal, e da manipulação digital dessas imagens, o que favorece a identificação fiel do canal endocervical e do orifício interno, facilitando a realização da biometria cervical.

Towner et al. ${ }^{(\mathbf{1 4})}$, em trabalho publicado em 2004, reportaram que a medida do comprimento do colo uterino aferido por US 3D é maior que na US 2D. De acordo com 
os pesquisadores, a média das medidas do colo aferidas por US 2D no corte sagital foi de $35,7 \mathrm{~mm}$, e a por US 3D foi de $43,7 \mathrm{~mm}$, sendo que em 21 das 34 pacientes examinadas o comprimento cervical foi maior que $5 \mathrm{~mm}$ ou mais no exame tridimensional do que no bidimensional, demonstrando diferença significante na comparação das médias entre os métodos bidimensional e tridimensional ${ }^{(\mathbf{1 4})}$. Esses pesquisadores relataram que a diferença entre os dois métodos de imagem é diretamente proporcional ao comprimento do colo uterino aferido por US 3D, ou seja, quanto maior o colo, maior a diferença entre essas técnicas. Segundo os autores, a medida do comprimento cervical em gestantes com colo uterino longo é subestimada pela US 2D. Towner et al. concluíram que a aferição da biometria da cérvice por exame ultra-sonográfico bidimensional é incompleta, justificando o baixo VPP atribuído ao método. O emprego da US 3D na avaliação do colo uterino no rastreamento do TPP pode ser útil; entretanto, relataram que se faz necessário o estudo de maior número de casos, que possam estabelecer critérios na aferição do comprimento cervical por US $3 \mathrm{D}^{(\mathbf{1 4})}$.

\section{TÉCNICA DO EXAME \\ CERVICAL TRANSVAGINAL TRIDIMENSIONAL}

Na realização da US 3D, os dados são provenientes de cortes consecutivos da imagem em 2D, originados da movimentação do transdutor ${ }^{(\mathbf{1 9})}$. O equipamento de ultra-som utilizado apresenta ambos os módulos, bidimensional e tridimensional, com sonda vaginal multifreqüencial de 5,0-9,0 $\mathrm{MHz}^{(19)}$.

Existem duas diferentes formas de captura das informações pelo equipamento de US no modo tridimensional ${ }^{(2,19)}$. No modo externo ("free-hand"), os dados são capturados por sondas comuns, utilizadas no exame bidimensional, por meio de movimento manual do transdutor, e transferidos via sinal de vídeo para serem elaborados e processados por um computador acoplado ao aparelho de $\mathrm{US}^{(2)}$. No modo interno ou de captura automática, a sonda utilizada é especial, e o sistema 3D responsável pelo processamento das informações é parte integrante do equipamento de ultra-som ${ }^{(2)}$. O transdutor possui, em seu interior, um motor que movimenta os cristais em diferentes planos, fazendo aquisições seqüenciais de imagens em 2D $\mathrm{D}^{(2)}$. No sistema "freehand", em especial, qualquer movimento brusco altera a reconstrução tridimensional, interferindo na qualidade da imagem $^{(19)}$. O desenvolvimento de sonda especial com mecanismo de varredura mecânica automática quadruplicou a velocidade de aquisição dos dados, aperfeiçoando a US 3D, e atualmente é o modo mais empregado $^{(2)}$.

Após a aquisição dos dados, que dura em média de 0,5 a 5 segundos, a partir da imagem bidimensional do colo no plano sagital, e independentemente do sistema utilizado, as informações são transferidas para a memória do equipamento, armazenadas e processadas, e as imagens exibidas simultaneamente no monitor em plano ortogonal (organização multiplanar) nos cortes axial, transverso e coronal, sendo possível a manipulação digital dessas imagens pelo examinador, favorecendo estudo detalhado do objeto e posteriormente a reconstrução tridimensional ${ }^{(\mathbf{1 9 , 2 0})}$.

Utilizando-se o sistema tridimensional, as estruturas podem ser medidas de forma linear em exposição multiplanar ou pode ser realizado cálculo volumétrico dos órgãos, mediante delineação manual do objeto de interesse exposto no monitor em organização multiplanar ou pelo sistema VOCAL (automático) ${ }^{(\mathbf{1 9})}$.

Na US 3D o comprimento cervical pode ser aferido em exposição multiplanar nos cortes sagital e coronal (Figura 2), bem como as medidas do afunilamento (diâme-

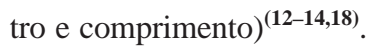

É importante ressaltar que a supremacia do método tridimensional em relação ao bidimensional no estudo do colo uterino se deve à possibilidade de manipulação digital das imagens em 3D, principalmente em colo com variação anatômica, o que permite a identificação exata da localização dos orifícios cervicais, com visualização clara do canal endocervical, aprimorando a aquisição do comprimento cervical.

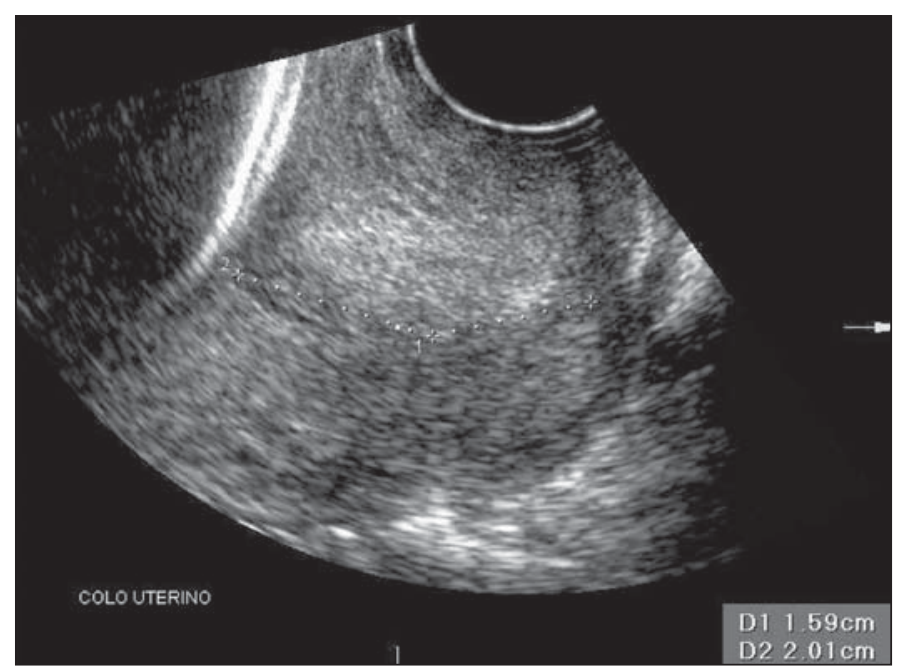

A

Figura 2. Imagem ultra-sonográfica tridimensional do colo uterino demonstrando os planos sagital e coronal. Figura representativa da técnica de aferição do comprimento cervical por US 3D.

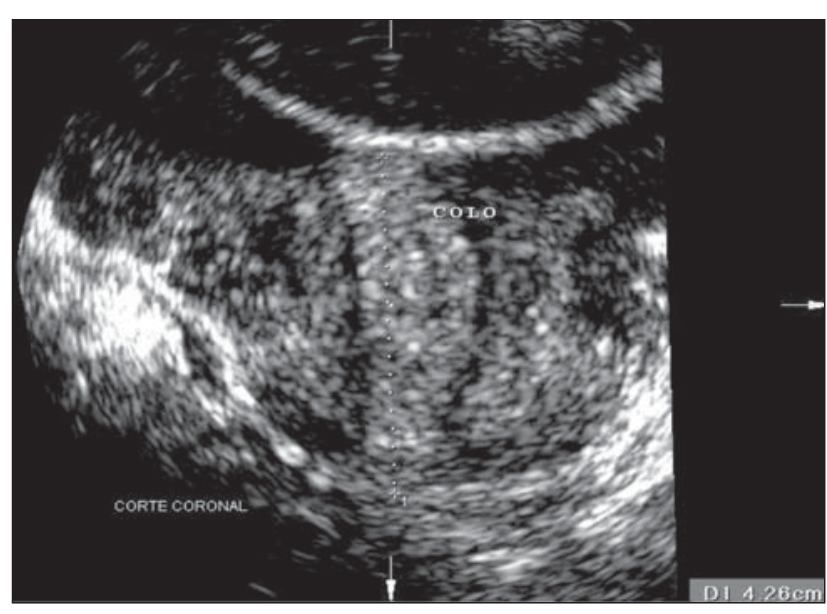

B 
Uma das grandes vantagens da US 3D é a capacidade de armazenamento das informações pelo aparelho de US em dispositivos eletrônicos, o que permite a reavaliação do mesmo exame por observador diferente ou a avaliação na ausência da paciente diante de diagnósticos duvidosos ${ }^{(\mathbf{2 0 , 2 1 )}}$.

\section{CONCLUSÕES}

Essa nova tecnologia abre perspectiva de acréscimo na acurácia da US cervical no rastreamento do trabalho de parto pré-termo, diminuindo resultados falso-negativos da US 2D, vez que, por meio da visualização tridimensional, o colo uterino pode ser mais bem explorado em face da possibilidade de diagnóstico do afunilamento, em casos supostamente normais, à luz da US $2 \mathrm{D}$, e auxilia a identificação precisa da trajetória do canal endocervical.

Diante das diferenças entre os métodos de exames bidimensional e tridimensional na aferição da biometria e da facilidade de análise da morfologia cervical com a tecnologia tridimensional, acreditamos que a US 3D do colo venha a acrescentar-se ao arsenal de exames atualmente utilizados na avaliação da cérvice no rastreamento do TPP, objetivando melhorar o prognóstico da prematuridade. Todavia, novas pesquisas com maior número de casos são necessárias na confirmação desses resultados.

\section{REFERÊNCIAS}

1. Szilard J. An improved three-dimensional display system. Ultrasonics 1974;12:273-276.

2. Maymon R, Herman A, Ariely S, Dreazen E, Buckovsky I, Weinraub Z. Three-dimensional vaginal sonography in obstetrics and gynecology. Human Reproduction Update 2000;6:475-484.

3. Tanaka Y, Okamura S, Doi S, et al. A preliminary report of computerized ultrasonography in obstetrics and gynecology: a new technique of C-mode. Nippon Sanka Fujinka Gakkai Zasshi 1982;34: 101-108.

4. Sarti DA, Sample WF, Hobel CJ, Staisch KJ. Ultrasonic visualization of a dilated cervix during pregnancy. Radiology 1979;130:417-420.

5. Varma TR, Patel RH, Pillai U. Ultrasonic assessment of cervix in normal pregnancy. Acta Obstet Gynecol Scand 1986;65:229-233.

6. Andersen HF, Nugent CE, Wanty SD, Hayashi RH. Prediction of risk for preterm delivery by ultrasonographic measurement of cervical length. Am J Obstet Gynecol 1990;163:859-867.

7. Iams JD, Paraskos J, Landon MB, Teteris JN, Johnson FF. Cervical sonography in preterm labor. Obstet Gynecol 1994;84:40-46.

8. Zorzoli A, Soliani A, Perra M, Caravelli E, Galimberti A, Nicolini U. Cervical changes throughout pregnancy as assessed by transvaginal sonography. Obstet Gynecol 1994;84:960-964.

9. Iams JD, Goldenberg RL, Meis PJ, et al. The length of the cervix and the risk of spontaneous premature delivery. National Institute of Child Health and Human Development Maternal Fetal Medicine Unit Network. N Engl J Med 1996; 334:567-572

10. Sonek J, Shellhaas C. Cervical sonography: a review. Ultrasound Obstet Gynecol 1998;11:71-78.

11. Zalar WR Jr. Early cervical length, preterm prelabor and gestational age at delivery. Is there a relationship? J Reprod Med 1998;43:1027-1033.

12. Hoesli IM, Surbek VD, Tercanli S, Holzgreve W. Three-dimensional volume measurement of the cervix during pregnancy compared to conventional 2D-sonography. Int J Gynaecol Obstet 1999;64:115-119.

13. Severi MF, Bocchi C, Florio P, Picciolini E D'Aniello G, Petraglia F. Comparison of two-dimensional and three-dimensional ultrasound in the assessment of the cervix to predict preterm delivery. Ultrasound Med Biol 2003;29:12611265 .

14. Towner D, Boe N, Lou K, Gilbert M. Cervical length measurements in pregnancy are longer when measured with three-dimensional transvaginal ultrasound. J Matern Fetal Neonatal Med 2004;16:167-170

15. Strauss A, Heer I, Fuchshuber S, Janssen U, Hillemanns P, Hepp H. Sonographic cervical volumetry in higher order multiple gestation. Fetal Diagn Ther 2001;16:346-353.

16. Strauss A, Muller-Egloff S, Heer IM, Dannecker C, Hepp H. Cervical incompetence in multifetal gestation: diagnosis and prophylaxis. Gynakol Geburtshilfliche Rundsch 2003;43:91-97.

17. Rozenberg P, Rafii A, Senat MV, Dujardin A Rapon J, Ville Y. Predictive value of two-dimensional and three-dimensional multiplanar ultrasound evaluation of the cervix in preterm labor. J Matern Fetal Neonatal Med 2003;13:237-241.

18. Bega G, Lev-Toaff A, Kuhlman K, et al. Threedimensional multiplanar transvaginal ultrasound of the cervix in pregnancy. Ultrasound Obstet Gynecol 2000;16:351-358.

19. Blaas HG, Eik-Nes SH, Berg S. Three-dimensional fetal ultrasound. Baillieres Best Pract Res Clin Obstet Gynaecol 2000;14:611-627.

20. Kratochwil A, Lee A, Schoisswohl A. Networking of three-dimensional sonography volume data. Ultrasound Obstet Gynecol 2000;16:335340 .

21. Chaoui R, Kalache KD, Hartung J. Application of three-dimensional power Doppler ultrasound in prenatal diagnosis. Ultrasound Obstet Gynecol 2001; 17:22-29. 